\title{
La Rúbrica en la Enseñanza Universitaria: Un Recurso Para la Tutoría de Grupos de Estudiantes
}

\author{
Manuela Raposo y Esther Martínez \\ Departamento de Didáctica, Organización Escolar y Métodos de Investigación de la Universidad \\ de Vigo, Facultad de Ciencias de la Educación, Campus As Lagoas s/n, 32004 Ourense-España \\ (e-mail: mraposo@uvigo.es; esthermf@uvigo.es)
}

Recibido Mar. 10, 2011; Aceptado Abr. 07, 2011; Versión final recibida May. 04, 2011

\begin{abstract}
Resumen
En este trabajo se presenta la experiencia de las autoras en el uso de la rúbrica para facilitar el proceso de tutoría de trabajos en grupo. La tutoría forma parte de las funciones asociadas a la docencia universitaria, y las tareas insertas en la función tutorial tales como el asesoramiento, la orientación y el seguimiento del trabajo del alumnado son complejas cuando se cuenta con un número elevado de estudiantes. La experiencia de las autoras con la rúbrica permite constatar que se trata de un instrumento útil que proporciona retroalimentación al alumnado durante las sesiones de tutoría y que le permite conocer de antemano las competencias y elementos que van a ser valorados junto con la puntuación otorgada. De este modo que junto con favorecer el proceso de enseñanza-aprendizaje, también lo hace al de la evaluación.
\end{abstract}

Palabras clave: rúbrica, tutoría, enseñanza universitaria, trabajo en grupo, recursos docentes

\section{The Rubric in University Education: A Resource for Mentoring Groups of Students}

\begin{abstract}
This paper presents the experience of the authors in using the rubric to facilitate the mentoring process of group work. Tutoring belongs to the functions associated with university teaching, and some of the tasks included in the tutorial work such as advice, guidance and monitoring of students' work are complex when the class has a large number of students. The authors experience working with the rubric shows that this is a useful tool that provides feedback to students during tutoring sessions and lets they know in advance the skills and elements to be used and the score given to their work. In this way, the method favors the teaching-learning process and also the evaluation of student's work.
\end{abstract}




\section{INTRODUCCIÓN}

En la renovación metodológica que está sufriendo la universidad española en su proceso de adaptación al Espacio Europeo de Educación Superior cobra especial relevancia el trabajo por competencias, particularmente las de tipo genérico o transversales, aquellas que son deseables con independencia de la titulación de origen, entre las que se encuentran el trabajo en grupo y la comunicación oral (González y Wagenaar, 2005). Para ello se ponen en juego diferentes estrategias y se enuncian actividades que, en la mayoría de los casos, necesitan de una orientación y seguimiento a través de la tutoría.

Se puede considerar el trabajo en grupo o en pequeños equipos, como una estrategia didáctica donde se distribuye la totalidad del alumnado en conjuntos que poseen un número limitado de personas definido según la finalidad de la tarea encomendada. Un grupo no es la suma o yuxtaposición de las partes sino la integración de sus efectivos, sus miembros trabajan juntos y de forma coordinada, orientados por un objetivo común encaminado a resolver las actividades propuestas y a desarrollar su propio aprendizaje. Para un buen funcionamiento se precisa de cierto grado de compromiso y confianza en las posibilidades del otro. Cada equipo tiene entidad propia dada por el tema que aborda, por el docente o por sus propios componentes. El punto final de un trabajo de este tipo es, en muchas ocasiones, su presentación oral al gran grupo, lo que exige de dos aspectos fundamentales: organizar un discurso específico para ser mostrado oralmente, teniendo en cuenta que está dirigido a una determinada audiencia y considerar su "puesta en escena", su exposición.

La tutoría forma parte de las funciones asociadas a la docencia universitaria; y las tareas insertas en la función tutorial como el asesoramiento, la orientación y el seguimiento del trabajo del alumnado son complejas cuando se cuenta con un número elevado de estudiantes para llevarla a cabo individualmente o en grupo, de forma presencial o virtual. Las competencias tutoriales que el docente necesita varían, según Ballesteros (2002, citado en Martínez y Raposo, 2010a), en función de que vayan dirigidas al grupo o al individuo, tal y como se muestra en la Tabla 1. Como se puede observar, el desarrollo de la acción tutorial en grupo precisa del dominio de un mayor número de competencias que en el caso de realizar una tutoría individual. Para poder dar respuesta a esta situación se elabora y se utiliza una rúbrica como recurso soporte del seguimiento realizado en una tarea que se resolvía en pequeños grupos, como se detalla más adelante.

Tabla 1: Competencias para la acción tutorial en grupo e individual (Ballesteros, 2002, citado en Martínez y Raposo, 2010a)

Competencias para la acción tutorial en grupo

Potenciar competencias de relación y comunicación.

Enseñar a tomar decisiones en grupo.

Desarrollar la práctica argumentativa en si mismo y en el alumnado.

Delegar la autogestión y autonomía en el grupo.

Utilizar estrategias de mediación en los conflictos.

Poseer recursos para la negociación.

Asumir la figura y rol de autoridad.

Considerar la justicia y equidad.

Competencias para la acción tutorial individual

Ajustar constantemente las necesidades psicoeducativas de cada alumno/a.

Lograr tiempos y espacios de diálogo e intercambio con cada alumno/a fuera del contexto de aula.

Escuchar activamente en las diferentes situaciones.

Una rúbrica puede considerarse como un instrumento en el que se definen criterios de valoración y diferentes estándares que se corresponden con niveles progresivos de ejecución de una tarea (Gil, 2007). Algunos autores coinciden en concebirla como una herramienta versátil que puede utilizarse de forma muy diferente para evaluar y para tutorizar los trabajos de los estudiantes (Bilbatua y Egizabal, 2010). Blanco (2008) se refiere a ellas como guías de puntuación que describen las características específicas del desempeño de un producto, proyecto o tarea en 
varios niveles de rendimiento o ejecución. Por su parte, Cebrián et al. (2007) la entienden como una herramienta válida para el proceso de enseñanza-aprendizaje que "ayuda a definir y explicar a los estudiantes lo que espera el profesor que aprendan, y dispone de criterios sobre cómo va a ser valorado su trabajo con ejemplos claros y concretos. (...) El alumno puede observar sus avances en términos de competencias, saber en cualquier momento qué le queda por superar y qué ha superado y cómo".

Por tanto, en el ámbito de la educación superior, la rúbrica se convierte tanto en una estrategia válida para la orientación y seguimiento del trabajo del alumnado, con identidad suficiente y autónoma al servicio de un determinado proceso formativo; como en una escala de valoración asociada a la evaluación, con entidad propia o bien, al servicio de otros instrumentos como puede ser el portafolio. De este modo, la rúbrica se presenta como un recurso para la evaluación integral y formativa, como un instrumento de orientación o como herramienta pedagógica. Se está entonces ante un instrumento que establece criterios con los que tutorizar, valorar y evaluar distintos niveles de desempeño y dominio de competencias, por ello a la rúbrica se le conoce también como plantilla de evaluación o matriz de valoración.

\section{LA RÚBRICA: UN RECURSO EN LA ENSEÑANZA UNIVERSITARIA}

Más allá del concepto de la rúbrica, Barberá y De Martín (2009) especifican su forma y procedimiento indicando que una rúbrica "se presenta como una pauta o tabla de doble entrada que permite unir y relacionar criterios de evaluación, niveles de logro y descriptores. La columna indica dimensiones de la calidad y enumera una serie de ítems o áreas que se deben evaluar. La fila indica los niveles de dominio. En la intersección se incluye una descripción textual de las cualidades de los resultados y productos en esa dimensión y a ese nivel". El formato que puede adoptar la rúbrica es una tabla de texto que puede estar en soporte estático (papel o archivo informático para consulta y descarga -.doc; .pdf; .gif) o dinámico (página web o software específico de creación y utilización de rúbricas), denominándose en este caso e-rúbrica y presentando determinadas características propias. Entre ellas, Cebrián (2007) destaca: más interacción; mejor autonomía por parte de los estudiantes para visionar las competencias adquiridas; más conocimiento y rapidez por parte del docente para detectar competencias difíciles de alcanzar por los grupos o individualmente; y más inmediatez en el proceso de comunicación y evaluación profesor-estudiante.

Como recurso para la evaluación integral y formativa (Conde y Pozuelo, 2007), se cree que con el uso de esta herramienta siempre se puede mejorar (Stiggins et al., 2005). La utilización de rúbricas, siguiendo a Kan (2007), permite la interiorización de los criterios de evaluación, promover la realización de actividades bajo criterios de calidad, así como la reflexión sobre el rendimiento y errores. Así, Andrade (2005) diferencia entre rúbricas de calificación (scoring rubrics) y rúbricas formativas (instructional rubrics). Para lograr que sean realmente formativas, el estudiante debe implicarse en todo el proceso de aprendizaje, autoevaluándose, evaluando a sus compañeros, coevaluando junto al profesor, e incluso llegando a participar en el propio diseño de la rúbrica (Fallas, 2005). Como instrumento de orientación y evaluación de la práctica educativa (Hafner y Hafner, 2003; Mertler, 2001; Moskal y Leydens, 2000; Tierney y Simon, 2004), se considera que con las rúbricas, los trabajos están siempre en progreso, así pueden funcionar bien para obtener una determinada información sobre el nivel curricular y de desempeño. Como herramienta pedagógica, se considera que la rúbrica favorece el aprendizaje significativo y el logro de competencias (Andrade, 2005; Fallas, 2005; Herrero y Torres, 2009). El estudiante, mediante este instrumento conoce claramente cuáles son las expectativas del docente, adquiere las pautas que le guiarán en la consecución de las competencias de aprendizaje y sitúa con precisión las dudas y problemas surgidos durante el proceso (Torres y Perera, 2010). Como indican Torres y Perera (2010: 142), "por una parte, se provee al alumnado de un referente que proporciona un feedback relativo a cómo mejorar su trabajo. Por otra, proporciona al profesor la posibilidad de manifestar sus expectativas sobre los objetivos de aprendizaje fijados".

Este último enfoque es el que orienta la práctica que se presenta en el uso de las rúbricas con estudiantes universitarios de segundo curso que realizan la carrera de Magisterio, con la intención de ser futuros docentes en la especialidad de Educación Infantil. 


\section{EXPERIENCIAS EN LA UTILIZACIÓN DE RÚBRICAS}

El origen del trabajo con las rúbricas en el contexto de la materia Nuevas Tecnologías aplicadas a la Educación viene dado por el momento de experimentación de los planes de estudio, con la adaptación de la titulación a los planteamientos del Espacio Europeo de Educación Superior y la consideración de las materias en créditos ECTS, de pronto, se tenía en el aula más de 80 estudiantes y dos docentes responsables. El contacto y conocimiento de experiencias realizadas en la Universidad de Málaga (Cebrián, 2007; 2008) fue el desencadenante para iniciarse en la construcción de la primera rúbrica para el portafolio de las prácticas de la citada materia (Raposo y Sarceda, 2008). En ella se presentaron los criterios de evaluación de objetivos acordes a las tareas propuestas en las prácticas y que, a su vez, responden a las finalidades y competencias establecidas en el diseño de la materia.

En el curso siguiente, teniendo en cuenta sus virtudes y los logros alcanzados en la experiencia desarrollada con los créditos prácticos de la materia, se selecciona aquel aspecto vinculado a la teoría donde, a priori, se creía que la rúbrica aportaría más beneficios, teniendo en cuenta las condiciones estructurales, organizativas y de contexto con las que se daría clase, siendo éste en un momento en el que convivían planes de estudio con y sin adaptar al proceso de Bolonia. Dicho aspecto fue el trabajo de ampliación y profundización de contenidos que debían realizar en pequeños grupos y que tenía un peso del $20 \%$ en la nota final, con una carga estimada de trabajo del estudiante de 25 horas que se desglosan del siguiente modo: 16 horas para la realización del trabajo (buscar información, leerla y seleccionarla, sintetizarla y organizarla para la elaboración del informe), 4 horas para la preparación, realización de la exposición y del material en soporte tecnológico que la acompaña) y 5 horas para la asistencia a las tutorías en grupo específicas sobre el trabajo.

En base a ello, se ha diseñado, utilizado y evaluado una rúbrica (Martínez y Raposo, 2009, 2010a, 2010b; Raposo y Martínez, 2010) que orienta al alumnado durante la realización de dicho trabajo en grupo y favorece su seguimiento en las sesiones de tutoría que recibe en la citada materia. Aspecto con más detalle a continuación.

\section{La rúbrica para el trabajo en grupo}

Para diseñar el recurso se tuvo en cuenta el Modelo Gavilán 2.0 (Gavilán, 2006) que estructura por fases los procesos fundamentales que están presentes en cualquier investigación. Así, en base al anterior, en éste se diferencian cinco fases en la elaboración del trabajo en equipo y cada una de ellas cuenta con su respectiva rúbrica que se emplea en las sesiones de tutoría. En la figura 1 se muestran los cinco momentos del trabajo en grupo donde cada fase tiene asociada su respectiva rúbrica.

Cada una de estas fases coincide con una sesión de tutoría grupal presencial en la que, tras escuchar lo que plantea el alumnado, orientar a éste y ofrecerle asesoramiento, tanto las docentes como los propios estudiantes aplican las rúbricas diseñadas a tal efecto, así se podría contrastar la información obtenida y revisar aquellos aspectos donde hay discrepancias. A continuación, se describen los componentes de cada una de las rúbricas que se utilizan en cada fase, aunque los niveles de desempeño varían de unas a otras.

Definición del tema: conociendo la trascendencia que tiene este primer momento de trabajo en grupo, con esta rúbrica se valora la claridad en la definición de la formulación del objetivo y propuesta de trabajo, la argumentación e interrogantes a resolver, junto con la actualidad, pertinencia y relevancia del tema.

Consulta de fuentes documentales: en esta fase del trabajo en grupo, la rúbrica debe constatar si los alumnos han hecho una adecuada consulta de diferente documentación. Se considera si son actuales, pertinentes y coherentes con el tema elegido, si aportan credibilidad, claridad o si son adecuadas, al mismo tiempo que se tiene en cuenta la internacionalización de las fuentes. 


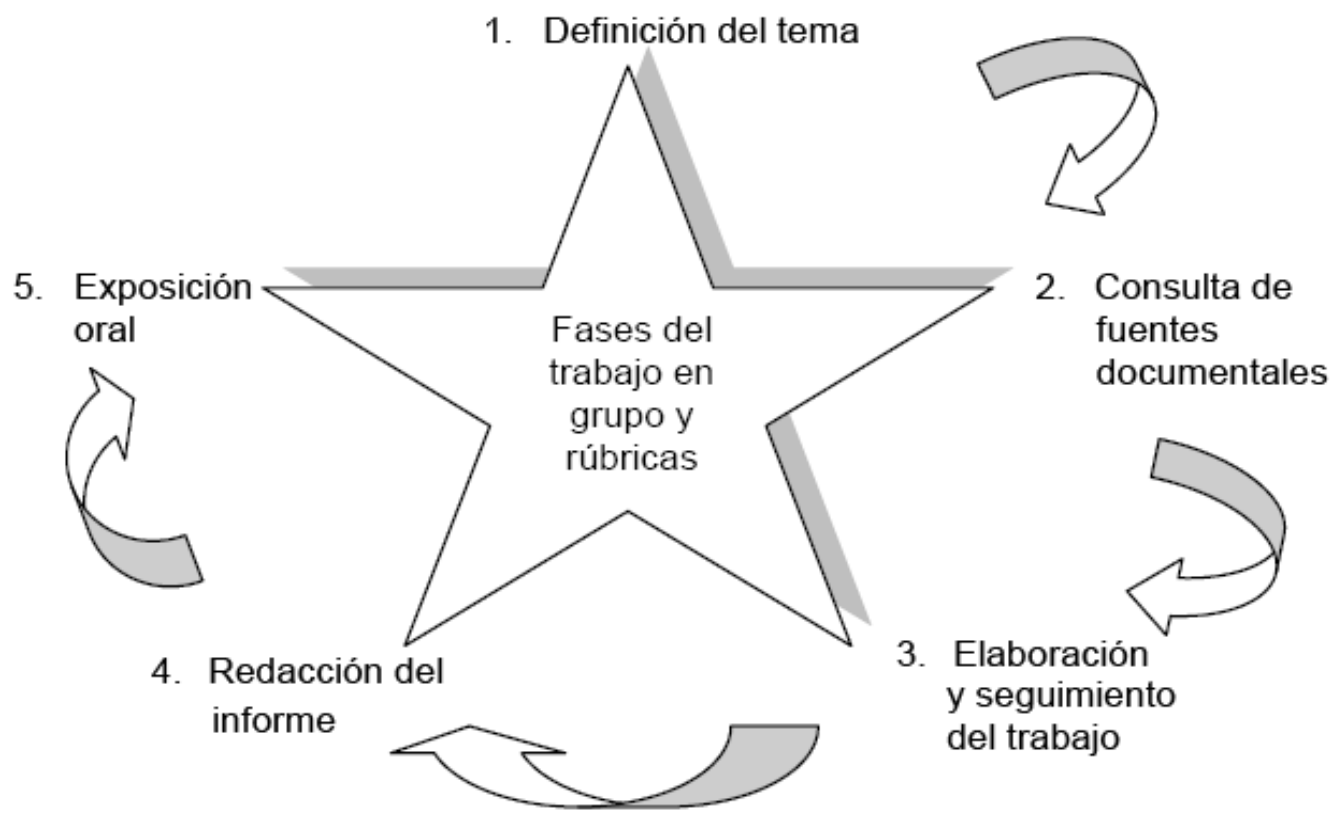

Fig. 1: Fases del trabajo en grupo y rúbricas asociadas

Elaboración y seguimiento del trabajo: aquí las docentes actúan como verdaderas guías del proceso. Por ello, la rúbrica diseñada sintetiza los elementos clave y presta atención a: que exista un índice completo de contenidos, las partes del trabajo estén coordinadas e integradas, tenga aplicación en la práctica profesional, si presentan dudas coherentes, si todo el grupo conoce todo el trabajo, la puntualidad en la entrega, el sentimiento de grupo, la responsabilidad en cada miembro y la interacción entre los miembros.

Redacción del informe de trabajo: esta rúbrica se basa en la propuesta realizada por Raposo y Sarceda (2008), considerando las siguientes dimensiones en el trabajo final de grupo: aspectos de formato (portada, numeración de páginas, tamaño de la letra, ortografía y claridad de redacción); contenido (introducción correcta, reflexión sobre el proceso, fuentes documentales bien referenciadas, apartados compensados, tamaño del trabajo equilibrado, si es completo y el contenido de todos los apartados está bien desarrollado) y actitud hacia el trabajo (seriedad en la entrega y soporte digital).

Exposición oral: siendo la culminación del trabajo en grupo, esta rúbrica tiene en cuenta aspectos como: la comunicación en la presentación (volumen de voz, seguridad, contacto visual con la audiencia, lenguaje gestual, postura corporal, vocabulario apropiado, discurso estructurado, ritmos adecuados, gestión de los tiempos, no utiliza apoyos escritos y el discurso no es monótono), el conocimiento del trabajo (dominio completo del tema, contesta con precisión a las preguntas, demuestra capacidad de síntesis), el uso adecuado del tiempo, discurso coherente, características y utilización del audiovisual empleado (aspectos técnicos correctos y válidos, atractivo, información no redundante, sobrecarga de información en la pantalla y si responde a una síntesis del trabajo).

Cada una de ellas se presenta al alumnado en formato Excel dentro de la sección de "Documentos y enlaces", que posee la plataforma de teleformación propia de la Universidad, denominada TEMA (http://faitic.uvigo.es/). Se opta por este formato porque permite el sumatorio y las ponderaciones totales de parciales en los criterios asignados (Raposo y Martínez, 2010). La Tabla 2 muestra la rúbrica utilizada para la exposición oral, valorada con un máximo de 3 puntos sobre 10 (Raposo y Martínez, 2010). 
Tabla 2: Rúbrica utilizada para la exposición oral

\begin{tabular}{|c|c|c|c|c|}
\hline \multirow{22}{*}{ 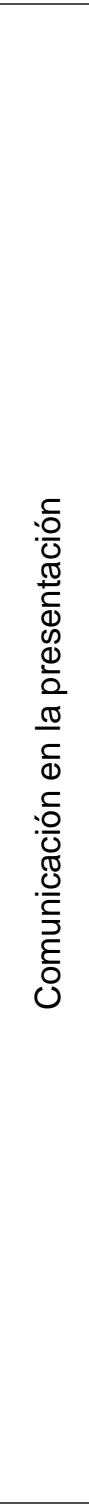 } & \multirow{2}{*}{\multicolumn{2}{|c|}{$\begin{array}{l}\text { El volumen de voz no es ni alto ni claro para } \\
\text { poder ser escuchado. } \\
0\end{array}$}} & \multicolumn{2}{|c|}{$\begin{array}{c}\text { El volumen de voz es alto y claro para poder ser } \\
\text { escuchado. }\end{array}$} \\
\hline & & & \multicolumn{2}{|c|}{0.1} \\
\hline & $\begin{array}{l}\text { Su voz no demuestra } \\
\text { seguridad. }\end{array}$ & \multicolumn{2}{|c|}{$\begin{array}{l}\text { Su voz demuestra a veces } \\
\text { seguridad. }\end{array}$} & $\begin{array}{l}\text { Su voz demuestra siempre } \\
\text { seguridad. }\end{array}$ \\
\hline & 0 & \multicolumn{2}{|c|}{0.05} & 0.1 \\
\hline & $\begin{array}{l}\text { No establece contacto visual } \\
\text { con la audiencia. }\end{array}$ & \multicolumn{2}{|c|}{$\begin{array}{l}\text { Establece algunas veces } \\
\text { contacto visual con la audiencia. }\end{array}$} & $\begin{array}{l}\text { Establece siempre contacto visual } \\
\text { con la audiencia. }\end{array}$ \\
\hline & 0 & \multicolumn{2}{|c|}{0.05} & 0.1 \\
\hline & $\begin{array}{l}\text { No utiliza buen lenguaje } \\
\text { gestual. }\end{array}$ & \multicolumn{2}{|c|}{$\begin{array}{l}\text { Utiliza algunas veces buen } \\
\text { lenguaje gestual. }\end{array}$} & $\begin{array}{c}\text { Utiliza siempre buen lenguaje } \\
\text { gestual. }\end{array}$ \\
\hline & 0 & \multicolumn{2}{|c|}{0.05} & 0.1 \\
\hline & $\begin{array}{l}\text { No adopta buena postura } \\
\text { corporal. }\end{array}$ & \multicolumn{2}{|c|}{$\begin{array}{l}\text { Adopta algunas veces buena } \\
\text { postura corporal. }\end{array}$} & $\begin{array}{c}\text { Adopta siempre buena postura } \\
\text { corporal. }\end{array}$ \\
\hline & 0 & \multicolumn{2}{|c|}{0.05} & 0.1 \\
\hline & $\begin{array}{l}\text { No utiliza un vocabulario } \\
\text { apropiado. }\end{array}$ & \multicolumn{2}{|c|}{$\begin{array}{l}\text { Utiliza a veces un vocabulario } \\
\text { apropiado. }\end{array}$} & $\begin{array}{l}\text { Utiliza siempre un vocabulario } \\
\text { apropiado. }\end{array}$ \\
\hline & 0 & \multicolumn{2}{|c|}{0.05} & 0.1 \\
\hline & $\begin{array}{l}\text { No utiliza un discurso } \\
\text { estructurado. }\end{array}$ & \multicolumn{2}{|c|}{$\begin{array}{l}\text { Utiliza a veces un discurso } \\
\text { estructurado. }\end{array}$} & $\begin{array}{l}\text { Utiliza siempre un discurso } \\
\text { estructurado. }\end{array}$ \\
\hline & 0 & \multicolumn{2}{|c|}{0.05} & 0.1 \\
\hline & No utiliza ritmos adecuados. & \multicolumn{2}{|c|}{$\begin{array}{l}\text { Utiliza a veces ritmos } \\
\text { adecuados. }\end{array}$} & Utiliza siempre ritmos adecuados. \\
\hline & 0 & \multicolumn{2}{|c|}{0.05} & 0.1 \\
\hline & $\begin{array}{l}\text { No gestiona bien los } \\
\text { tiempos. }\end{array}$ & \multicolumn{2}{|c|}{$\begin{array}{l}\text { Gestiona a veces bien los } \\
\text { tiempos. }\end{array}$} & $\begin{array}{l}\text { Gestiona siempre bien los } \\
\text { tiempos. }\end{array}$ \\
\hline & 0 & \multicolumn{2}{|c|}{0.05} & 0.1 \\
\hline & $\begin{array}{l}\text { Continuamente utiliza } \\
\text { apoyos escritos. }\end{array}$ & \multicolumn{2}{|c|}{$\begin{array}{c}\text { En ocasiones utiliza apoyos } \\
\text { escritos. }\end{array}$} & No utiliza apoyos escritos. \\
\hline & 0 & \multicolumn{2}{|c|}{0.05} & 0.1 \\
\hline & \multicolumn{2}{|c|}{ El discurso es monótono. } & \multicolumn{2}{|c|}{ El discurso no es monótono. } \\
\hline & \multicolumn{2}{|c|}{0} & \multicolumn{2}{|c|}{0.1} \\
\hline \multirow{6}{*}{ 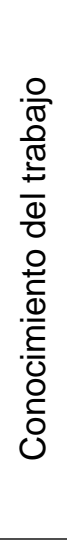 } & $\begin{array}{l}\text { No parece conocer bien el } \\
\text { tema. }\end{array}$ & \multicolumn{2}{|c|}{$\begin{array}{l}\text { Demuestra un conocimiento de } \\
\text { partes del tema. }\end{array}$} & $\begin{array}{l}\text { Demuestra un conocimiento } \\
\text { completo del tema. }\end{array}$ \\
\hline & 0 & \multicolumn{2}{|c|}{0.15} & 0.25 \\
\hline & $\begin{array}{l}\text { No es capaz de contestar } \\
\text { con precisión a las } \\
\text { preguntas planteadas. }\end{array}$ & \multicolumn{2}{|c|}{$\begin{array}{l}\text { Es capaz de contestar con } \\
\text { precisión a algunas preguntas } \\
\text { planteadas. }\end{array}$} & $\begin{array}{l}\text { Es capaz de contestar con } \\
\text { precisión a todas las preguntas } \\
\text { planteadas. }\end{array}$ \\
\hline & 0 & \multicolumn{2}{|c|}{0.15} & 0.25 \\
\hline & $\begin{array}{l}\text { No demuestra capacidad de } \\
\text { síntesis en aquellos } \\
\text { aspectos que lo precisan. }\end{array}$ & $\begin{array}{r}\text { Demuestra a } \\
\text { capacidad d } \\
\text { aquellos asp } \\
\text { prec }\end{array}$ & $\begin{array}{l}\text { Inas veces } \\
\text { íntesis en } \\
\text { tos que lo } \\
\text { n. }\end{array}$ & $\begin{array}{l}\text { Demuestra siempre capacidad de } \\
\text { síntesis en aquellos aspectos que } \\
\text { lo precisan. }\end{array}$ \\
\hline & 0 & 0. & & 0.25 \\
\hline & $\begin{array}{l}\text { Utiliza el tiempo } \\
\text { inadecuadamente. }\end{array}$ & $\begin{array}{r}\text { Utiliza el tiem } \\
\text { quedan algul } \\
\text { descomp }\end{array}$ & $\begin{array}{l}\text { bien pero } \\
\text { aspectos } \\
\text { sados. }\end{array}$ & Utiliza el tiempo adecuadamente. \\
\hline$\stackrel{\omega}{=}$ & 0 & 0 & & 0.15 \\
\hline$\underset{\Phi}{\alpha}$ & $\begin{array}{l}\text { No utiliza un discurso } \\
\text { coherente. }\end{array}$ & $\begin{array}{l}\text { Utiliza algun } \\
\text { discurso c }\end{array}$ & $\begin{array}{l}\text { veces un } \\
\text { erente. }\end{array}$ & $\begin{array}{l}\text { Utiliza siempre un discurso } \\
\text { coherente. }\end{array}$ \\
\hline & 0 & 0. & & 0.1 \\
\hline
\end{tabular}


Tabla 2: (continuación)

\begin{tabular}{|c|c|c|c|c|}
\hline \multirow{13}{*}{ 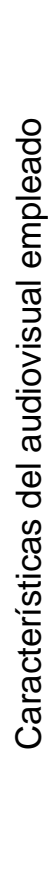 } & $\begin{array}{l}\text { No utiliza adecuadamente } \\
\text { los audiovisuales para } \\
\text { apoyar su explicación. }\end{array}$ & \multicolumn{2}{|c|}{$\begin{array}{l}\text { Utiliza algunas veces } \\
\text { adecuadamente los } \\
\text { audiovisuales para apoyar su } \\
\text { explicación. }\end{array}$} & $\begin{array}{l}\text { Utiliza siempre adecuadamente } \\
\text { los audiovisuales para apoyar su } \\
\text { explicación. }\end{array}$ \\
\hline & 0 & \multicolumn{2}{|c|}{0.1} & 0.15 \\
\hline & $\begin{array}{l}\text { Pocos aspectos técnicos } \\
\text { (color, tamaño y tipo de } \\
\text { letra, imágenes...) son } \\
\text { correctos y adecuados. }\end{array}$ & \multicolumn{2}{|c|}{$\begin{array}{c}\text { Algunos aspectos técnicos } \\
\text { (color, tamaño y tipo de letra, } \\
\text { imágenes...) son correctos y } \\
\text { adecuados. }\end{array}$} & $\begin{array}{l}\text { Todos los aspectos técnicos } \\
\text { (color, tamaño y tipo de letra, } \\
\text { imágenes...) son correctos y } \\
\text { adecuados. }\end{array}$ \\
\hline & 0.1 & \multicolumn{2}{|c|}{0.15} & 0.25 \\
\hline & \multicolumn{2}{|c|}{ El audiovisual no es atractivo. } & \multicolumn{2}{|c|}{ El audiovisual es atractivo. } \\
\hline & \multicolumn{2}{|l|}{0} & \multicolumn{2}{|r|}{0.1} \\
\hline & \multicolumn{2}{|c|}{ La información presentada es redundante. } & \multicolumn{2}{|c|}{ La información presentada no es redundante. } \\
\hline & \multicolumn{2}{|l|}{0} & \multicolumn{2}{|r|}{0.1} \\
\hline & \multicolumn{2}{|c|}{$\begin{array}{l}\text { Hay una sobrecarga de información por } \\
\text { pantalla. }\end{array}$} & \multicolumn{2}{|c|}{$\begin{array}{l}\text { No hay una sobrecarga de información por } \\
\text { pantalla. }\end{array}$} \\
\hline & \multicolumn{2}{|c|}{0} & \multicolumn{2}{|c|}{0.1} \\
\hline & \multicolumn{2}{|c|}{ No responde a una síntesis del trabajo. } & \multicolumn{2}{|c|}{ Responde a una síntesis del trabajo } \\
\hline & \multirow{2}{*}{\multicolumn{2}{|c|}{$\frac{0}{\text { Puntuación Máxima· } 3 \text { nuntos }}$}} & \multicolumn{2}{|r|}{0.2} \\
\hline & & & \multicolumn{2}{|r|}{ Total: } \\
\hline
\end{tabular}

\section{DISCUSIÓN}

El uso de la rúbrica supone que el alumnado conoce de antemano las competencias y elementos que van a ser valorados junto con la puntuación otorgada, y viceversa, cuando un alumno/a ve una determinada puntuación conoce los criterios en base a los cuales se le ha atribuido. En este sentido, se realiza una evaluación para el aprendizaje entendida en los términos que formulan Benito y Cruz (2005: 88): "la evaluación para el aprendizaje es el proceso de buscar e interpretar evidencias para que estudiantes y profesores conozcan dónde se encuentra el alumno en relación a su aprendizaje, dónde necesita estar y cuál es el modo mejor de llegar allí".

Este instrumento no sólo favorece el proceso de enseñanza-aprendizaje porque actúa de guía; sino también el de evaluación pues permite objetivizar cualquier trabajo de los alumnos y restarle el componente de subjetividad que pudiera tener el evaluador (Martínez y Raposo, 2010). Cuando la materia es compartida por varios docentes, según constatan Raposo y Sarceda (2008), el disponer de una rúbrica permite ser más coherentes entre ellos a la hora de emitir un juicio de valor, así como asegurar a cada alumno/a que va a ser evaluado con los mismos criterios que sus compañeros/as.

Así, si interesa dotar a este recurso de una finalidad evaluadora, cabe señalar que con la utilización de la rúbrica desde el comienzo del proceso, el alumnado conoce los criterios con los que va a ser evaluado junto con las exigencias asociadas a una competencia específica, lo que le puede permitir regular y orientar su aprendizaje (Raposo y Sarceda, 2008). Realizar trabajos en grupo y exposiciones orales son estrategias metodológicas habituales en las aulas universitarias y recomendables para el desarrollo de competencias de carácter general.

\section{CONCLUSIONES}

La experiencia desarrollada con el uso de la rúbrica en la orientación y seguimiento de trabajos en grupo pone de manifiesto una serie de conclusiones que se convierten en bondades de este recurso. 
1.- Se trata de un instrumento útil para proporcionar retroalimentación al alumnado durante las sesiones de tutoría, por disponer de información detallada sobre su nivel de desempeño en una determinada tarea.

2.- Permite que la atención y seguimiento de un número elevado de trabajos sea mejor al tener constancia escrita de los detalles y toma de decisiones.

3.- El uso de las rúbricas durante las sesiones de tutorización de trabajos de grupo necesitó de un trabajo previo exhaustivo para la definición de los criterios y aspectos a considerar en cada fase del proceso.

4.- La puesta en práctica ha demostrado que entre las rúbricas y las tutorías se produce una relación interdependiente y bidireccional, ya que durante las tutorías se matizó el contenido de las rúbricas para hacerlo más accesible y comprensible por el alumnado.

5.- El instrumento acota y clarifica el marco general de la tarea a realizar, ayuda en la orientación y seguimiento y centra las conversaciones del grupo con las docentes.

\section{REFERENCIAS}

Andrade, H. G., Teaching with rubrics: The good, the bad, and the ugly, College Teaching, 53 (1), 27-30 (2005).

Barberá, E. y De Martín, E., Portfolio electrónico: aprender a evaluar el aprendizaje, Editorial UOC, Barcelona (2009).

Benito, A. y Cruz, A., Nuevas claves para la docencia universitaria en el Espacio Europeo de Educación Superior, Narcea, Madrid -España (2005).

Bilbatua, M. y Egizabal, D. Las rúbricas en la reflexión sobre el trabajo en equipo., en Bujan, K. (coord.): Seminario internacional Las rúbricas de evaluación en el desempeño de competencias: ámbitos de investigación y docencia, Universidad del País Vasco, San Sebastián (España) 17-18 de junio (2010).

Blanco Blanco, A., Las rúbricas: un instrumento útil para la evaluación de competencias, en La enseñanza universitaria centrada en el aprendizaje: estrategias útiles para el profesorado por L. Prieto (coord.), 171-188, Octaedro-ICE de la Universidad de Barcelona, Barcelona (2008).

Cebrián, M., Buenas prácticas en el uso del e-portafolio y e-rúbrica, In El practicum: buenas prácticas en el Espacio Europeo de Educación Superior por A. Cid, M. Raposo y A. Pérez (coords.), 67-87, Tórculo, Santiago de Compostela (2007).

Cebrián, M., La evaluación formativa mediante e-rúbricas, INDIVISA - Boletín de Estudios e Investigación-. Monografía X, 197-208 (2008).

Cebrián, M., Raposo, M. y Accino, J., E-portfolio en el practicum: un modelo de rúbrica, Comunicación y Pedagogía: 218, 8-13 (2007).

Conde, A. y Pozuelo, F., Las plantillas de evaluación (rúbrica) como instrumento para la evaluación. Un estudio de caso en el marco de la reforma de la enseñanza universitaria en el EEES, Investigación en la Escuela: 63, 77-90 (2007).

Fallas, I. V., El uso de rúbricas para la evaluación de cursos en línea. Conferencia Internacional de Educación a Distancia, San Juan Puerto Rico, agosto (2005).

Gavilán, Modelo Gavilán 2.0: una propuesta para el desarrollo de la competencia para manejar información (CMI), 2006, http://www.eduteka.org/pdfdir/ModeloGavilan.php. Acceso: 17 Abril (2011). 
Gil Flores, J., La evaluación de competencias laborales, Revista Educación XXI, 10, 83-106 (2007)

González, J. y Wagenaar, R., Tuning Educational Structures in Europe: universities' constitution to the Bologna process (en línea), 2005. Bilbao: Publicaciones de la Universidad de Deusto. http://www.tuning.unideusto.org/tuningeu/index.php?option=com_docman\&ltemid=59\&task=view_ category\&catid=19\&order=dmdate_published\&ascdesc=DESC. Acceso: 18 de Abril (2011).

Hafner, J.C. y Hafner, P.M., Quantitative analysis of the rubric as an assessment tool: an empirical study of student peer-group rating, International J. Science Education: 25 (12), 1509-1528 (2003).

Herrero, E. A. y Torres, J. J., Aplicación de un nuevo instrumento pedagógico en la adquisición de competencias: la rúbrica en la docencia de Geometría Descriptiva, In Estrategias de innovación en el nuevo proceso de evaluación del aprendizaje por I. Rodríguez (Ed.), 273-284, Servicio de publicaciones de la Universidad Europea Miguel de Cervantes (2009).

Kan, A., An alternative method in the new educational program from the point of performancebased assessment. Rubric scoring scales, Educational Sciences: Theory \& Practice: 7 (1), 144152 (2007).

Martínez, M.E. y Raposo, M. La rúbrica como recurso en la tutoría: percepciones del alumnado, en Bujan, K. (coord.): Seminario internacional Las rúbricas de evaluación en el desempeño de competencias: ámbitos de investigación y docencia, Universidad del País Vasco, San Sebastián (España) 17-18 de junio (2010a).

Martínez, M.E. y Raposo, M. La rúbrica en la tutorización de trabajos en grupo: opiniones y valoraciones, IV Jornadas de Innovación Didáctica, Universidad de Vigo, Vigo (España), 11 de diciembre (2009).

Martínez, M.E. y Raposo, M., Seguimiento de trabajos tutelados en grupo mediante rúbricas, In La docencia en el nuevo escenario del EEES, por Vicerrectoría de Formación e Innovación Educativa, 567-570, Universidad de Vigo, Vigo (España) (2010b).

Mertler, C. A., Designing scoring rubrics for your classroom, Practical Assessment, Research \& Evaluation, ISSN: 1531-7714 (en línea), 7 (25),1-9 (2001)

Moskal, B.M. y Leydens, J.A., Scoring rubric development: validity and reliability, Practical Assessment, Research \& Evaluation, 7 (10), 1-9 (2000).

Raposo, M. y Sarceda, M. C. Como avaliar unha memoria de prácticas? Un exemplo de rúbrica no ámbito das novas tecnoloxías, eln Prácticas educativas innovadoras na universidade, 107-124, Universidad de Vigo, Vigo (España) (2008).

Raposo, M.y Martínez, M.E. Un ejemplo de rúbrica en la plataforma Tema, en La innovación educativa en el contexto actual de la educación superior, editado por la Vicerrectoría de Formación e Innovación Educativa, 769-775, Universidad de Vigo, Vigo (España) (2010).

Roblyer, M.D. y Wiencke, W.R., Design and use of a rubric to assess and encourage interactive qualities in distance courses, The American Journal of Distance Education: 17 (2), 77-97 (2003).

Stiggins J.R., Arter J.A., Chappuis J. y Chappuis S., Classroom Assessment for Student Learning. Doing it right-using it well, $3^{\mathrm{a}}$ ed., Portland, Oregón, USA: Assessment Training Institute, Inc. (2005).

Tierney, R. y Simon, M., What's still wrong with rubrics: focusing on the consistency of performance criteria across scale levels. Practical Assessment, Research \& Evaluation, 9(2), 1-10 (2004).

Torres, J. J. y Perera, V. H. La rúbrica como instrumento pedagógico para la tutorización y evaluación de los aprendizajes en el foro online en educación superior. Píxel-Bit. Revista de Medios y Educación, 36, 141-149 (2010) 
